Check for updates

Cite this: RSC Adv., 2017, 7, 33248

\title{
Hydrotropic polymer-based paclitaxel-loaded self-assembled nanoparticles: preparation and biological evaluation
}

\author{
Lipeng Gao, ${ }^{a}$ Liefang Gao, ${ }^{a}$ Mingxue Fan, ${ }^{a}$ Qilong Li, ${ }^{a}$ Jiyu Jin, ${ }^{a}$ Jing Wang, ${ }^{a}$ \\ Weiyue Lu, ${ }^{b}$ Lei Yu, ${ }^{a}$ Zhiqiang Yan (D) *a and Yiting Wang*a
}

\begin{abstract}
The poor compatibility of carrier materials with drugs is one of the main obstacles in the drug encapsulation of nano-drug delivery system (NDDS), hindering the clinical translation of NDDS. In this study, using paclitaxel (PTX) as the insoluble model drug, we conjugated $N, N$-diethylniacinamide (DENA), a hydrotropic agent of PTX, to the backbone of poly(L- $\gamma$-glutamyl-glutamine) (PGG), a water-soluble polymer, to prepare the "hydrotropic polymer" PGG-DENA to improve its compatibility with PTX. By virtue of the hydrotropic effect of the DENA group, PTX was encapsulated by PGG-DENA to obtain the hydrotropic polymeric nanoparticles (PGG-DENA/PTX NPS). PTX-conjugated poly(L- $\gamma$-glutamyl-glutamine) acid (PGG-PTX) NPs previously reported were used as the control in the study. The PGG-DENA/PTX NPs showed a $z$-average hydrodynamic diameter of about $70 \mathrm{~nm}$, and good long-term stability in PBS solution at $4{ }^{\circ} \mathrm{C}$. The cumulative release rate of PTX from PGG-DENA/PTX NPs reached $79.10 \%$ at $96 \mathrm{~h}$, while that of PGG-PTX NPs was $22.96 \%$. PGG-DENA/PTX NPs showed significantly increased in vitro cytotoxicity on $\mathrm{NCl}-\mathrm{H} 460$ lung cancer cells compared with PGG-PTX NPs. The hemolysis study proved that the PGG-DENA/PTX NPs has good biocompatibility. These results indicated that by introducing the hydrotropic agent DENA, the hydrotropic polymer PGG-DENA becomes an effective carrier material of PTX. This study provides a solution to increase the compatibility of carrier materials with insoluble drugs, and also may provide an effective way to develop a series of personalized carrier materials suitable for different insoluble drugs.
\end{abstract}

Received 23rd April 2017 Accepted 26th June 2017 DOI: 10.1039/c7ra04563h rsc.li/rsc-advances
Accordingly, researchers introduced hydrotropic agents into the hydrophobic side of the polymeric materials to increase the drug encapsulation in micelles. ${ }^{3-5}$ Hydrotropic agents are a kind of small molecules that can increase the water solubility of insoluble drugs by forming a complex, association or complex salt. ${ }^{6}$ The micelles developed by combining the hydrotropic agents with the traditional micelles are so-called hydrotropic polymer micelles. Up to now, there have been several hydrotropic agents reported, such as $N$-methylpyridinium nicotinamide (PNA), ${ }^{7} \quad N, N$-dimethylbenzamide $\quad(\mathrm{DMBA}),{ }^{4} \quad \mathrm{~N}, \mathrm{~N}$ diethylniacinamide (DENA) $)^{3,4}$ and fluorenylmethoxycarbonyl $(\alpha-$ Fmoc). ${ }^{8}$ For example, Kim group developed a PEG- $b$-(poly- $N, N-$ diethylnicotinamide) (PEG- $b$-PDENA), which can self-assemble to form micelles that showed good encapsulating effect for PTX. ${ }^{4}$ The solubility of PTX was increased by 6000 times, which is higher than the equivalent concentration of free DENA or PEG- $b$-PLA does. The PTX encapsulated PEG- $b$-PDENA micelles can be stably stored for at least 4 weeks. ${ }^{9}$ Because DENA has a special solubilization mechanism for PTX (aromatic ring buildup and hydrogen bonding), it showed higher solubilization effect on PTX than other cosolvents (DMBA or $\alpha$-Fmoc) did. ${ }^{4}$ More importantly, different form the free hydrotropic agents, the polymerized hydrotropic agent cannot be easily taken up in vivo, thereby avoiding the potential toxicity to body. In addition, 
the stability and drug release behavior of micelles can be altered by adjusting the molecular weight of the hydrotropic end. ${ }^{4}$ In spite of this, the hydrotropic polymer micelles are also deficient: the hydrophobicity of the polymerized hydrotropic agents is weaker than that of the hydrophobic end of traditional micellar materials (such as PLA), especially for DENA. This resulted in a high critical micelle concentration (CMC) of these hydrotropic polymers, which is disadvantageous for the formation and further use of micelles. ${ }^{2}$ This problem hinders the further development of the hydrotropic polymer micelles.

PTX-conjugated poly(L-glutamic acid) (PGA-PTX, also known as Xyotax ${ }^{\mathrm{TM}}$ ), a polymer drug conjugate, has entered the clinical trials due to its high water solubility and antitumor effect. ${ }^{\mathbf{1 0 , 1 1}}$ Poly(L- $\gamma$-glutamyl-glutamine)-paclitaxel (PGG-PTX) is another polymer drug conjugate we previously developed on the basis of PGA-PTX, ${ }^{12-14}$ which further increased the water solubility, increased the tolerable dose, reduced the side effects and improved the antitumor effects. ${ }^{15-18}$ The results showed that PGG-PTX could be directly dissolved in water and had a solubility of $50 \mathrm{mg} \mathrm{mL}^{-1}$, which was much higher than that of PGAPTX (7 mg mL $\left.{ }^{-1}\right)$. It can self-assemble to form nano core-shell structure about $30 \mathrm{~nm}$ in water, with PTX as hydrophobic nucleus and PGG skeleton as hydrophilic shell. However, PGGPTX is also deficient: since PTX is covalently coupled to the PGG backbone, its drug release performance is poor. The cumulative release rate of PTX from PGG-PTX nanoparticles (NPs) in vitro is only about $23 \%$ at 96 h. $^{12}$

Based on the above considerations, we here conjugated DENA to the backbone of PGG to prepare the "hydrotropic polymer" PGG-DENA. Then by virtue of the hydrotropic effect of DENA group, PTX was encapsulated by PGG-DENA to obtain the hydrotropic polymeric nanoparticles (PGG-DENA/PTX NPs) (Fig. 1). We characterized the NPs by DLS, TEM, HPLC and evaluated the PTX release, cytotoxicity, long-term stability and

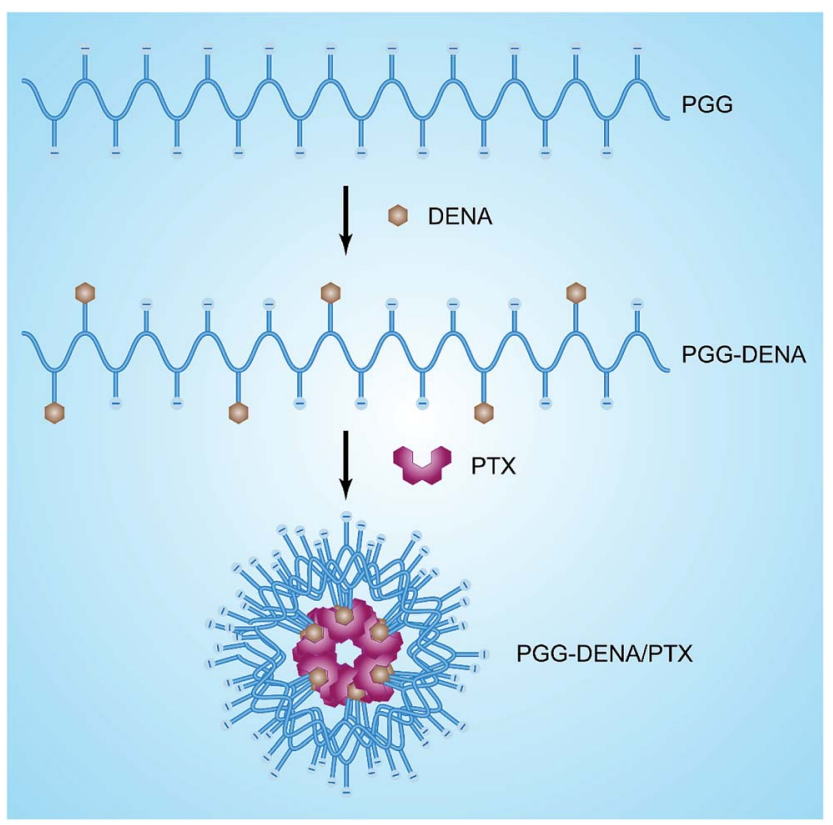

Fig. 1 Schematics of the preparation process of the PGG-DENA/PTX cellular uptake by human NCI-H460 cancer cells in vitro and hemolysis study in vivo. PGG-DENA/PTX NPs exhibited a desirable drug release profile, and good biocompatibility and longterm stability.

\section{Materials and methods}

\subsection{Materials}

PGG (poly-(L- $\gamma$-glutamyl-glutamine)) was synthesized by our laboratory. ${ }^{12}$-(Chloromethyl)benzoyl chloride, tert-butyldimethylsilyl chloride (TBSCL), 2-hydroxynicotinic acid, $N, N^{\prime}$-carbonyldiimidazole (CDI) and 4-dimethylaminopyridine (DMAP) were purchased from Sigma-Aldrich, Inc. $N$-(3-Dimethylaminopropyl)- $N$-ethylcarbodiimide (EDC) was purchased from EMD Chemicals Inc. (Darmstadt, Germany). All other chemicals and reagents were commercially available and directly used.

Human NCI-H460 carcinoma cell line was obtained from Shanghai Institute of Cell Biology. This cell was cultured in RPMI 1640 supplemented with $10 \%$ FBS at $37{ }^{\circ} \mathrm{C}$ in a humidified atmosphere of $5 \% \quad \mathrm{CO}_{2}$ and $95 \%$ air. DiO (3,3'-dioctadecyloxacarbocyanine, perchlorate) was purchased from Tianjin Biolite Biotech Co., LTD. Hoechst 33342 was purchased from Beyotime Institute of Biotechnology.

All experiments involving animals were performed in accordance with the guidelines of the Institutional Animal Care and Use Committee (IACUC) of East China Normal University. All experimental protocols were approved by the IACUC of East China Normal University. Male SD rats (6-8 weeks old) were obtained from SLAC Ltd (Shanghai, China) and maintained under SPF conditions. All efforts were made to minimize the number of animals used and their suffering. Animal experiments were reported in accordance with the ARRIVE (Animal Research: Reporting In Vivo Experiments) guidelines.

\subsection{Synthesis of PGG-DENA}

The synthesis of PGG-DENA was illustrated in Fig. 2. The starting material 4-(chloromethyl)benzoyl chloride (1) was reacted with boron hydride to prepare compound 2 , which was protected with TBSCl to afford compound 3. The starting material 2-hydroxynicotinic acid (4) was reacted with diethylamine to produce compound $\mathbf{5}$. The compound 3 was reacted with compound 5 to obtain compound $\mathbf{6}$, which was deprotected to afford compound 7. Then the compound 7 was conjugated to PGG in the presence of EDC and DMAP to obtain the final "hydrotropic polymer" PGG-DENA, which was dialyzed with a tangential flow filtration system followed by lyophilization.

2.2.1 Synthesis of (4-(chloromethyl)phenyl)methanol (2). The 4-(chloromethyl)benzoyl chloride (1) (1.34 g, $6 \mathrm{mmol}$ ) was dissolved in $100 \mathrm{~mL}$ THF and ethanol $(1: 1, \mathrm{v} / \mathrm{v})$. Then $\mathrm{NaBH}_{4}$ $(0.908 \mathrm{~g}, 24 \mathrm{mmol})$ was added and stirred at room temperature under $\mathrm{N}_{2}$ for $2 \mathrm{~h}$. The solvent was removed by rotary evaporation at $37{ }^{\circ} \mathrm{C}$. Ethyl acetate was added and washed with $0.3 \mathrm{M}$ $\mathrm{NaHCO}_{3}(\mathrm{aq})$. The organic layer was dried over $\mathrm{Na}_{2} \mathrm{SO}_{4}$, filtrated and concentrated to give (4-(chloromethyl)phenyl)methanol (2) (867 mg, 92\%) as white solid. ${ }^{1} \mathrm{H}-\mathrm{NMR}\left(400 \mathrm{MHz}, \mathrm{CDCl}_{3}\right) \delta 4.50$ 

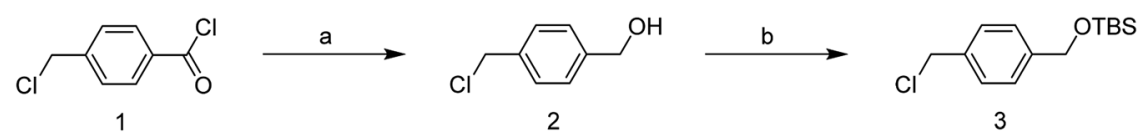<smiles>O=C(Cl)c1cccnc1O</smiles>

4

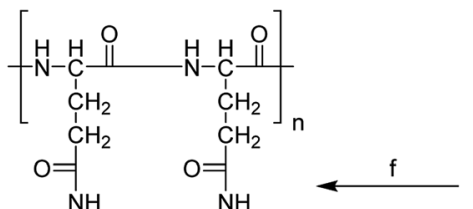

$\mathrm{HC}-\mathrm{COONa} \mathrm{HC}^{\mathrm{I}}-\mathrm{COONa}$

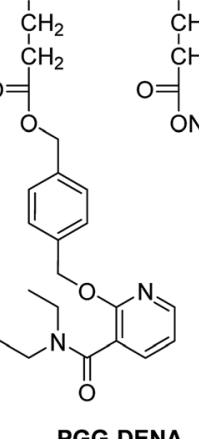<smiles>CCN(CC)C(=O)c1cccnc1O</smiles>

5

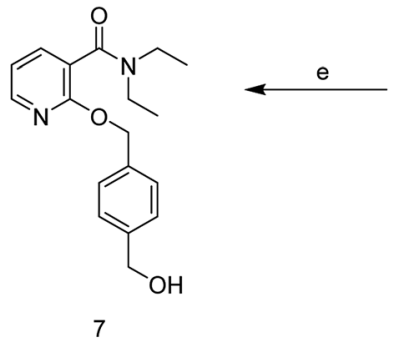<smiles>CC(C)C</smiles><smiles>CCCCOCc1ccc(COc2ncccc2C(=O)N(CC)CC)cc1</smiles>

6

Fig. 2 Synthesis of PGG-DENA. Reagents and conditions: (a) $\mathrm{NaBH}_{4}, \mathrm{THF} / \mathrm{EtOH}$, rt; (b) TBSCl, DMAP, DCM, rt; (c) DEA, CDI, THF, 60 ${ }^{\circ} \mathrm{C}$; (d) $\mathrm{K}_{2} \mathrm{CO}_{3}$, anhydrous acetone, $60{ }^{\circ} \mathrm{C}$; (e) $2 \mathrm{M} \mathrm{HCl}(\mathrm{aq})$, rt; (f) $\mathrm{PGG}-\mathrm{COOH}, \mathrm{EDC}$, DMAP, DMF, rt.

$(\mathrm{d}, J=4.8 \mathrm{~Hz}, 2 \mathrm{H}), 4.75(\mathrm{~s}, 2 \mathrm{H}), 5.21(\mathrm{t}, J=4.8 \mathrm{~Hz}, 1 \mathrm{H}), 7.32(\mathrm{~d}, J$ $=7.6 \mathrm{~Hz}, 2 \mathrm{H}), 7.39(\mathrm{~d}, J=7.6 \mathrm{~Hz}, 2 \mathrm{H})$.

2.2.2 Synthesis of tert-butyl((4-(chloromethyl)benzyl)oxy) dimethylsilane (3). Imidazole (95 mg, $1.4 \mathrm{mmol}$ ), (4-(chloromethyl)phenyl)methanol (2) (157 $\mathrm{mg}, 1 \mathrm{mmol})$ and DMAP ( $85 \mathrm{mg}, 0.7 \mathrm{mmol}$ ) were dissolved in $15 \mathrm{~mL}$ of $\mathrm{CH}_{2} \mathrm{Cl}_{2}$. TBSCl (256 mg, $1.7 \mathrm{mmol}$ ) was added and stirred at room temperature under $\mathrm{N}_{2}$ for $1 \mathrm{~h}$. Then, $50 \mathrm{~mL} \mathrm{KHSO}_{4}$ solution $(1 \mathrm{M})$ was added into the reaction solution, followed by extraction with $\mathrm{CH}_{2} \mathrm{Cl}_{2}$. The $\mathrm{CH}_{2} \mathrm{Cl}_{2}$ layer was collected and the solvent was removed by rotary evaporation and dried under vacuum to obtain tertbutyl((4-(chloromethyl)benzyl)oxy)dimethylsilane (3) (262 mg, 97\%) as clear oil. ${ }^{1} \mathrm{H}-\mathrm{NMR}\left(400 \mathrm{MHz}, \mathrm{CDCl}_{3}\right) \delta 7.34(\mathrm{~m}, 4 \mathrm{H}), 4.75$ $(\mathrm{s}, 2 \mathrm{H}), 4.59(\mathrm{~s}, 2 \mathrm{H}), 0.95(\mathrm{~s}, 9 \mathrm{H}), 0.11(\mathrm{~s}, 6 \mathrm{H})$.

2.2.3 Synthesis of $\mathrm{N}, \mathrm{N}$-diethyl-2-hydroxynicotinamide (5). The 2-hydroxynicotinic acid (4) (1 g, $7.2 \mathrm{mmol})$ was dissolved in $30 \mathrm{~mL}$ THF. CDI (1.17 g, $7.22 \mathrm{mmol})$ was added and stirred at $60{ }^{\circ} \mathrm{C}$ under $\mathrm{N}_{2}$ for $1 \mathrm{~h}$. Then, diethylamine ( $\left.0.79 \mathrm{~g}, 10.8 \mathrm{mmol}\right)$ was added to the system at room temperature, and the mixture was stirred at $60{ }^{\circ} \mathrm{C}$ under $\mathrm{N}_{2}$ for $2 \mathrm{~h}$. After the reaction system was cooled to room temperature, the solvent was removed by rotary evaporation. $10 \mathrm{~mL}$ ethyl acetate was added. The suspension was filtered through sintered glass funnel with EA to give $N, N$-diethyl-2-hydroxynicotinamide (5) $(1.18 \mathrm{~g}, 85 \%)$ as white solid. ${ }^{1} \mathrm{H}-\mathrm{NMR}\left(400 \mathrm{MHz}, \mathrm{DMSO}-d_{6}\right) \delta 11.84(\mathrm{~s}, 1 \mathrm{H}), 7.42$ $(\mathrm{dd}, J=6.5,1.9 \mathrm{~Hz}, 1 \mathrm{H}), 7.39(\mathrm{dd}, J=6.7,2.0 \mathrm{~Hz}, 1 \mathrm{H}), 6.21(\mathrm{t}, J=$
$6.6 \mathrm{~Hz}, 1 \mathrm{H}), 3.37(\mathrm{q}, J=7.0 \mathrm{~Hz}, 2 \mathrm{H}), 3.13(\mathrm{q}, J=7.0 \mathrm{~Hz}, 2 \mathrm{H}), 1.09$ (t, $J=7.1 \mathrm{~Hz}, 3 \mathrm{H}), 1.02(\mathrm{t}, J=7.1 \mathrm{~Hz}, 3 \mathrm{H})$.

2.2.4 Synthesis of 2-((4-((tert-butyldimethylsilyl)oxy) methyl)benzyl)oxy)- $N, N$-diethylnicotinamide (6). $N, N$-Diethyl-2hydroxynicotinamide (5) $(252.2 \mathrm{mg}, 1.3 \mathrm{mmol})$, tert-butyl((4(chloromethyl)benzyl)oxy)dimethylsilane (3) (271 mg, $1 \mathrm{mmol})$ and $\mathrm{K}_{2} \mathrm{CO}_{3}(414 \mathrm{mg}, 3 \mathrm{mmol}$ ) were dissolved in $20 \mathrm{~mL}$ anhydrous acetone. The reaction mixture was stirred at $60{ }^{\circ} \mathrm{C}$ under $\mathrm{N}_{2}$ for $24 \mathrm{~h}$, and then cooled to room temperature. Then $0.3 \mathrm{M}$ $\mathrm{NaHCO}_{3}(\mathrm{aq})$ was added to the reaction solution, which was extracted with EA $(3 \times 30 \mathrm{~mL})$. The organic layer was dried over $\mathrm{Na}_{2} \mathrm{SO}_{4}$, filtrated and concentrated to give 2-((4-(( tert-butyldimethylsilyl)oxy)methyl)benzyl)oxy)- $N, N$-diethylnicotinamide (6) (304 mg, 71\%) as white solid. ${ }^{1} \mathrm{H}-\mathrm{NMR}\left(400 \mathrm{MHz}, \mathrm{CD}_{3} \mathrm{OD}\right) \delta 7.79$ (dd, $J=6.7,1.4 \mathrm{~Hz}, 1 \mathrm{H}), 7.50(\mathrm{dd}, J=6.8,1.4 \mathrm{~Hz}, 1 \mathrm{H}), 7.30(\mathrm{~s}$, $4 \mathrm{H}), 6.42(\mathrm{t}, J=6.8 \mathrm{~Hz}, 1 \mathrm{H}), 5.21(\mathrm{~s}, 2 \mathrm{H}), 4.72(\mathrm{~s}, 2 \mathrm{H}), 3.51(\mathrm{q}, J=$ $7.1 \mathrm{~Hz}, 2 \mathrm{H}), 3.22(\mathrm{q}, J=7.1 \mathrm{~Hz}, 2 \mathrm{H}), 1.22(\mathrm{t}, J=7.1 \mathrm{~Hz}, 3 \mathrm{H}), 1.09$ (t, $J=7.2 \mathrm{~Hz}, 3 \mathrm{H}), 0.93(\mathrm{~s}, 9 \mathrm{H}), 0.08(\mathrm{~s}, 6 \mathrm{H})$.

2.2.5 Synthesis of $N, N$-diethyl-2-((4-(hydroxymethyl)benzyl) oxy)nicotinamide (7, DENA). The 2-((4-(( tert-butyldimethylsilyl) oxy)methyl)benzyl)oxy)- $N, N$-diethylnicotinamide (6) (1 g, 2.33 mmol) was dissolved in $20 \mathrm{~mL}$ THF. Then, the $\mathrm{pH}$ of the reaction mixture was adjusted to $2-3$ by $2 \mathrm{M} \mathrm{HCl} \mathrm{(aq)} \mathrm{and} \mathrm{stirred} \mathrm{at}$ room temperature for $1 \mathrm{~h}$. The solvent was removed by rotary evaporation. $30 \mathrm{~mL}$ DCM was added. The organic layer was washed with $0.3 \mathrm{M} \mathrm{NaHCO}_{3}(\mathrm{aq})(3 \times 30 \mathrm{~mL})$ and dried with 
$\mathrm{Na}_{2} \mathrm{SO}_{4}$. The organic layer was concentrated by rotary evaporation to give $N, N$-diethyl-2-((4-(hydroxymethyl)benzyl)oxy) nicotinamide (7) (652 mg, 89\%) as white solid. ${ }^{1} \mathrm{H}-\mathrm{NMR}(400$ $\left.\mathrm{MHz}, \mathrm{D}_{2} \mathrm{O}\right) \delta 7.75(\mathrm{~d}, J=6.8 \mathrm{~Hz}, 1 \mathrm{H}), 7.58(\mathrm{~d}, J=5.5 \mathrm{~Hz}, 1 \mathrm{H}), 7.29$ (d, $J=7.9 \mathrm{~Hz}, 2 \mathrm{H}), 7.19$ (d, $J=7.9 \mathrm{~Hz}, 2 \mathrm{H}), 6.52(\mathrm{t}, J=6.8 \mathrm{~Hz}$, $1 \mathrm{H}), 5.15(\mathrm{~s}, 2 \mathrm{H}), 4.52(\mathrm{~s}, 2 \mathrm{H}), 3.40(\mathrm{q}, J=7.1 \mathrm{~Hz}, 2 \mathrm{H}), 3.12(\mathrm{q}, J=$ $7.0 \mathrm{~Hz}, 2 \mathrm{H}), 1.11$ (t, $J=7.2 \mathrm{~Hz}, 3 \mathrm{H}), 0.94(\mathrm{t}, J=7.1 \mathrm{~Hz}, 3 \mathrm{H})$.

2.2.6 Synthesis of PGG-DENA. PGG (2.62 g, $16.5 \mathrm{mmol} /$ monomer-unit of polymer) was stirred in $30 \mathrm{~mL}$ anhy DMF for $30 \mathrm{~min}$. Then EDC $(2.53 \mathrm{~g}, 13.2 \mathrm{mmol})$, DMAP $(0.42 \mathrm{~g}, 3.4 \mathrm{mmol})$ and DENA ( 7 ) (2.51 g, $8 \mathrm{mmol})$ were added into the reaction and stirred at room temperature for $24 \mathrm{~h}$. The reaction was quenched by $0.3 \mathrm{M} \mathrm{NaHCO}_{3}(\mathrm{aq})$. The solution was stirred for $15 \mathrm{~min}$ and dialyzed (MWCO 10k) for $24 \mathrm{~h}$ against water, lyophilized to obtain PGG-DENA as a white solid. Then, the ${ }^{1} \mathrm{H}$ NMR spectra of PGG and PGG-DENA in deuterated water $\left(\mathrm{D}_{2} \mathrm{O}\right)$ were recorded with a Bruker spectrometer at $400 \mathrm{MHz}$. The $M_{\mathrm{w}}$ of PGG and PGG-DENA were characterized by gel permeation chromatography with a GPC-MALS system (Wyatt, Santa Barbara, California).

\subsection{Preparation of PGG-DENA/PTX NPs}

PGG-DENA/PTX NPs were prepared by the emulsificationsolvent evaporation method. ${ }^{\mathbf{1 9 , 2 0}}$ Briefly, $12 \mathrm{mg}$ of PTX was dissolved in $2 \mathrm{~mL}$ of mixture of methylene chloride and acetone ( $3: 1, \mathrm{v} / \mathrm{v}$ ) as the organic phase, and $40 \mathrm{mg}$ of PGG-DENA were suspended in $4 \mathrm{~mL}$ of sodium cholate solution as the water phase. The mixture was emulsified by ultrasonic method in ice bath and gently stirred at room temperature. The solution was then opened to air overnight to allow slow evaporation of organic solvent and formation of the PGG-DENA/PTX NPs, which were purified by G50 gel column connecting AKTA purifier (GE Healthcare) to remove the free PTX.

\subsection{PTX solubility}

The solubility of PTX was determined by high performance liquid chromatography (HPLC, Agilent 1260 series). DENA was dissolved in deionized water and packed in centrifuge tube (2 $\mathrm{mL}$ ). Excess PTX was added to each tube. The mixture was stirred using a magnetic stirring bar for $24 \mathrm{~h}$ at $37{ }^{\circ} \mathrm{C}$. The sample was mixed with $1 \mathrm{~mL}$ ethyl acetate to extract PTX. The ethyl acetate solution was dried with nitrogen blowing instrument. Then the samples were resuspended with acetonitrile and filtered through a $0.22 \mu \mathrm{m}$ pore-sized filtration membrane. The amount of PTX in each tube was measured by HPLC with a UV detector at $228 \mathrm{~nm}$.

\subsection{Characterization of PGG-DENA/PTX NPs}

The particle sizes of PGG-DENA/PTX NPs were measured by dynamic light scattering (DLS) using a Mastersizer2000 (Malvern Instruments Inc) equipped with He-Ne laser (4 mW, 633 $\mathrm{nm}$ ) light source and $90^{\circ}$ angle scattered-light collection configuration.

The particle charge was quantified as zeta potential using a Mastersizer2000 (Malvern Instruments Inc).
The morphology of PGG-DENA/PTX NPs were observed with transmission electron microscopy (TEM). The TEM study was carried out using a JEM-2100 (Hitachi, Tokyo, Japan) electron microscope operating at an accelerating voltage of $75 \mathrm{kV}$.

The drug loading capacity of PGG-DENA/PTX was determined by HPLC (Agilent 1260 series). PGG-DENA/PTX (4 mg) was placed in a $5 \mathrm{~mL}$ centrifuge tube. Then, $2 \mathrm{~mL}$ of acetonitrile was added and the solution was shaken horizontally at 120 $\min ^{-1}$ with an incubator shaker (HZ-8812S, Scientific and Educational Equipment plant, China) at $37^{\circ} \mathrm{C}$. The supernatant solution was taken at $0.5 \mathrm{~h}, 3 \mathrm{~h}, 6 \mathrm{~h}, 9 \mathrm{~h}$ and $20 \mathrm{~h}$, respectively, and filtered through a $0.22 \mu \mathrm{m}$ pore-sized filtration membrane for HPLC determination.

\subsection{Long-term stability of PGG-DENA/PTX NPs}

PGG-DENA/PTX NPs solution $\left(2.0 \mathrm{mg} \mathrm{mL}^{-1}\right)$ was prepared in PBS. The particle size and polydispersity index (PDI) of the sample was monitored by DLS for 28 days.

\subsection{In vitro release of PTX from PGG-DENA/PTX NPs}

To investigate the in vitro release profile of PTX from PGGDENA/PTX NPs, we used sodium salicylate solution $(0.8 \mathrm{M}, \mathrm{pH}$ 6.5) as the release medium as reported previously. ${ }^{21}$ The PGGDENA/PTX NPs solution with the final concentration of $2 \mathrm{mg}$ $\mathrm{mL}^{-1}$ was packed in $50 \mathrm{~mL}$ centrifuge tube and shaken horizontally at $120 \mathrm{~min}^{-1}$ at $37{ }^{\circ} \mathrm{C}$. The sample was withdrawn at predetermined time points and mixed with $1 \mathrm{~mL}$ ethyl acetate to extract PTX. The ethyl acetate solution was dried with nitrogen blowing instrument. Then the samples were resuspended with acetonitrile and filtered through a $0.22 \mu \mathrm{m}$ pore-sized filtration membrane. The medium was refreshed at various time intervals. The concentration of PTX was determined by HPLC analysis.

\subsection{In vitro cytotoxicity assays}

The in vitro cytotoxicity of NPs was investigated by the CCK-8 assay according to the published protocols with modifications. ${ }^{22}$ NCI-H460 cells $\left(5 \times 10^{3}\right)$ were seeded in 96-well plates and incubated for $24 \mathrm{~h}$ in a humidified atmosphere with $5 \%$ $\mathrm{CO}_{2}$. Then serial dilutions of PTX, PGG-PTX NPs, PGG-DENA/ PTX NPs and PGG-DENA were added to the plate $(100 \mu \mathrm{L}$ per well), respectively. After further incubation for up to $48 \mathrm{~h}$, the cells were treated with $10 \mu \mathrm{L}$ of CCK-8 solution and cultured for $4 \mathrm{~h}$. The absorbance was measured with a microplate reader (SpectraMax M5, Molecular Devices, USA) at $450 \mathrm{~nm}$. The survival rate was calculated using the following formula: viability rate $=\left[\left(\mathrm{OD}_{\text {test }}\right.\right.$ group $\left.-\mathrm{OD}_{\text {blank }}\right) /\left(\mathrm{OD}_{\text {control }}\right.$ group $\left.\left.\mathrm{OD}_{\text {blank }}\right)\right] \times 100 \%$, where $\mathrm{OD}_{\text {test group }}$ is the optical density (OD) of experiment group, $\mathrm{OD}_{\text {control group }}$ is the $\mathrm{OD}$ of control group, and $\mathrm{OD}_{\text {blank }}$ is the OD of blank group.

\subsection{In vitro cellular uptake}

2.9.1 Preparations of PGG-DENA/PTX/DiO NPs. PGGDENA/PTX/DiO NPs were prepared by the emulsificationsolvent evaporation method. ${ }^{19}$ Briefly, DiO was suspended in 
methylene chloride and acetone $(3: 1, \mathrm{v} / \mathrm{v})$ as the organic phase, and PGG-DENA/PTX NPs were suspended in sodium cholate solution as the water phase. The mixture was emulsified by ultrasonic method in ice bath and rotary evaporated to remove organic solvent and obtain the PGG-DENA/PTX/DiO NPs. PGGDENA/PTX/DiO NPs were then purified by G50 gel column connecting AKTA purifier to remove free DiO.

2.9.2 Cellular uptake. Cellular uptake studies were performed on NCI-H460 cell line. Cells were seeded in glass bottom dish or 6-well plates at a density of $3 \times 10^{4}$ cells per $\mathrm{mL}$ and cultured for $24 \mathrm{~h}$. Cells were washed with PBS and treated with different concentrations of PGG-PTX/DiO and PGG-DENA/PTX/ DiO NPs for $2 \mathrm{~h}$.

In order to observe the cellular uptake of NPs qualitatively, the treated cells were washed three times with PBS, then fixed with $4 \%$ paraformaldehyde, stained with Hoechst 33342 and observed using a confocal laser scanning microscope (CLSM, TCS SP5, Leica).

To quantitatively analyze the cellular uptake of NPs, the treated cells were washed with PBS, trypsinized and harvested by centrifugation at $1200 \mathrm{rpm}$ for $5 \mathrm{~min}$. The cells were resuspended in $200 \mu \mathrm{L}$ PBS and filtered through a $40 \mathrm{~mm}$ nylon mesh

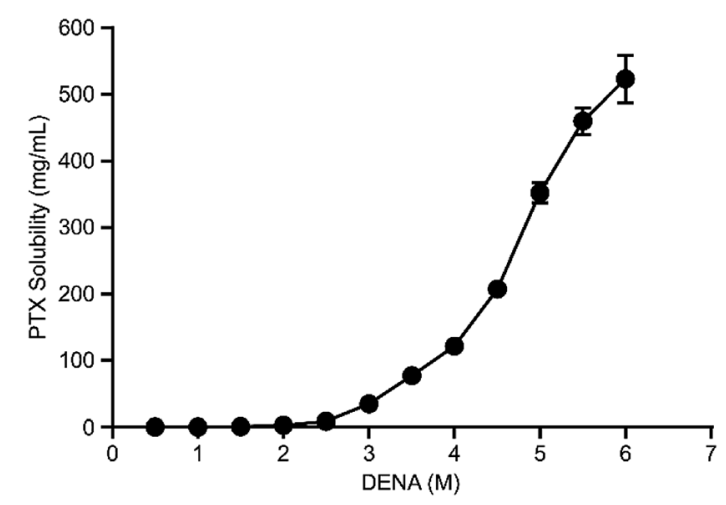

Fig. 3 The solubility of PTX as a function of the molar concentration of DENA. The solubility of PTX reached about $523 \mathrm{mg} \mathrm{mL}^{-1}$ at $6 \mathrm{M}$ of DENA. Data are means \pm SD from three measurements. to remove cell aggregates. The cell suspensions were then analyzed by flow cytometry (Guava easyCyte, USA).

\subsection{Hemolysis study}

Hemolysis study was carried out according to the published procedure. ${ }^{23}$ Briefly, freshly collected rat blood was washed three times with saline by centrifugation at $1500 \mathrm{rpm}$ for 15 minutes. The red blood cell suspension was diluted with saline to obtain a $2 \%$ suspension $(\mathrm{v} / \mathrm{v})$. Various concentrations of PGGDENA/PTX NPs, Cremophor EL, Tween 80, saline were added into the suspension, respectively. These samples were incubated at $37{ }^{\circ} \mathrm{C}$ for 1 hour, and centrifuged at $3000 \mathrm{rpm}$ for 10 minutes. The supernatants were collected and analyzed for hemoglobin content by spectrophotometric detection at $545 \mathrm{~nm}$. Analysis of each sample was performed in triplicate.

\subsection{Statistical analysis}

Statistical differences were evaluated by two-tailed student's $t$ test for two groups of data and one-way ANOVA for over three groups of data. The differences were considered to be significant at $P<0.05$ and very significant at $P<0.01$.

\section{Results and discussion}

\subsection{PTX solubility}

The solubilization of PTX by DENA was determined by HPLC. The results (Fig. 3) showed that the solubility of PTX increased gradually as the concentration of DENA increased. When the concentration of DENA reached $6 \mathrm{M}$, the solubility of PTX was increased to about $523 \mathrm{mg} \mathrm{mL} \mathrm{m}^{-1}$ in water (the intrinsic solubility of PTX is $\left.0.0003 \mathrm{mg} \mathrm{mL}{ }^{-1}\right) .{ }^{24}$ The results indicating that DENA increased the solubility of PTX by $1.7 \times 10^{6}$-fold.

\subsection{Characterization of PGG-DENA}

3.2.1 Characterization of ${ }^{\mathbf{1}} \mathbf{H}$-NMR spectra. The chemical structure of the PGG-DENA is shown in Fig. 4A. The green part is PGG, and the red is DENA. The ${ }^{1} \mathrm{H}-\mathrm{NMR}$ spectra of PGG (I) and PGG-DENA (II) are shown in Fig. 4B. The characteristic peaks
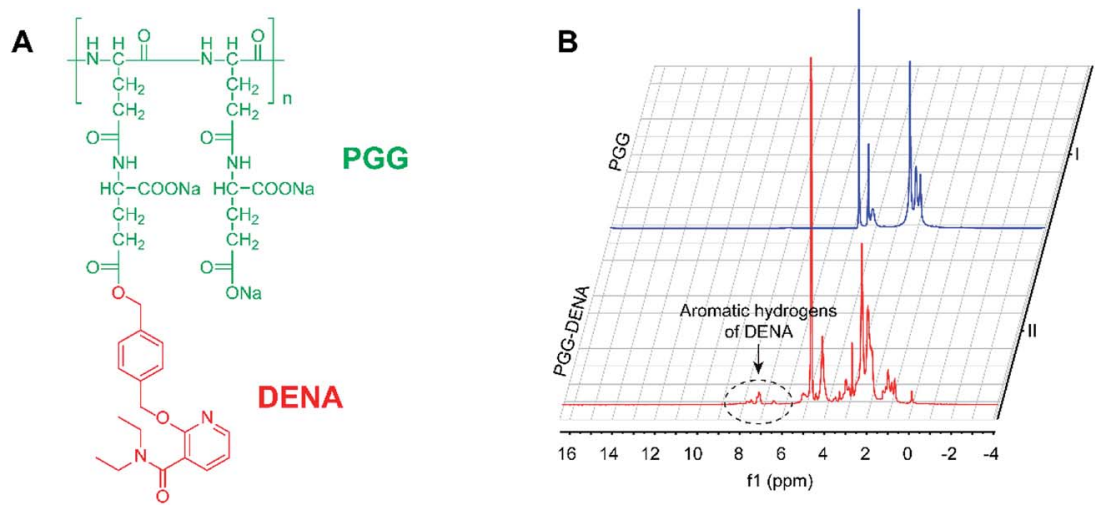

Fig. 4 Characterization of the PGG-DENA. (A) The chemical structure of the PGG-DENA. The green part is PGG and the red is DENA. (B) ${ }^{1} H-$ NMR spectra of PGG (I) and PGG-DENA (II). The characteristic peaks aromatic hydrogens of DENA at 7.80 (s, 1H), 7.59 (s, 1H), 7.21-7.30 (m, 4H) and $6.53(\mathrm{~s}, 1 \mathrm{H}) \mathrm{ppm}$ (the black arrow) showed that DENA and PGG were successfully linked together. 
aromatic hydrogens of DENA at $7.80(\mathrm{~s}, 1 \mathrm{H}), 7.59(\mathrm{~s}, 1 \mathrm{H}), 7.21-$ $7.30(\mathrm{~m}, 4 \mathrm{H})$ and $6.53(\mathrm{~s}, 1 \mathrm{H}) \mathrm{ppm}$ (the black arrow) can be found in the ${ }^{1} \mathrm{H}-\mathrm{NMR}$ spectrum of PGG-DENA. There was no peak found at 7-8 ppm in the spectra of PGG. The ${ }^{1} \mathrm{H}-\mathrm{NMR}$ results showed that DENA and PGG had been successfully linked together.

3.2.2 Characterization of $\boldsymbol{M}_{\mathbf{w}}$. The results are shown in Table 1. The $M_{\mathrm{w}}$ of PGG and PGG-DENA were about $51.29(\mathrm{kDa})$ and $65.52(\mathrm{kDa})$, and the PDI were 1.31 and 1.43, respectively. The PGG/DENA molar ratio was calculated to about $4.66: 1$. And the results also suggested the successful conjugated of DENA.

\subsection{Characterization of PGG-DENA/PTX NPs}

3.3.1 Characterization of DLS and TEM. The particle size of PGG-DENA/PTX NPs was measured by DLS method. The result (Fig. 5A) showed that the average particle size of PGG-DENA/ PTX NPs was about $70 \mathrm{~nm}$, with a PDI of 0.219. In addition, the zeta potential of the PGG-DENA/PTX NPs was $-30.3 \mathrm{mV}$. In order to observe the morphology of NPs, the particles were negatively stained with phosphotungstic acid and then visualized using TEM. PGG-DENA/PTX NPs (Fig. 5B) showed welldefined spherical morphology. The particle size of PGGDENA/PTX NPs observed by TEM was about $50 \mathrm{~nm}$, which was smaller than that determined by DLS. We speculated that the

Table 1 Characterization of polymer conjugates ${ }^{a}$

\begin{tabular}{lll}
\hline Sample & $M_{\mathrm{w}}(\mathrm{kDa})$ & $M_{\mathrm{w}} / M_{\mathrm{n}}(\mathrm{PDI})$ \\
\hline PGG & 51.29 & 1.31 \\
PGG-DENA & 65.52 & 1.43
\end{tabular}

${ }^{a}$ All data are expressed as the mean $M_{\mathrm{w}}$ of the samples $(n=3)$.
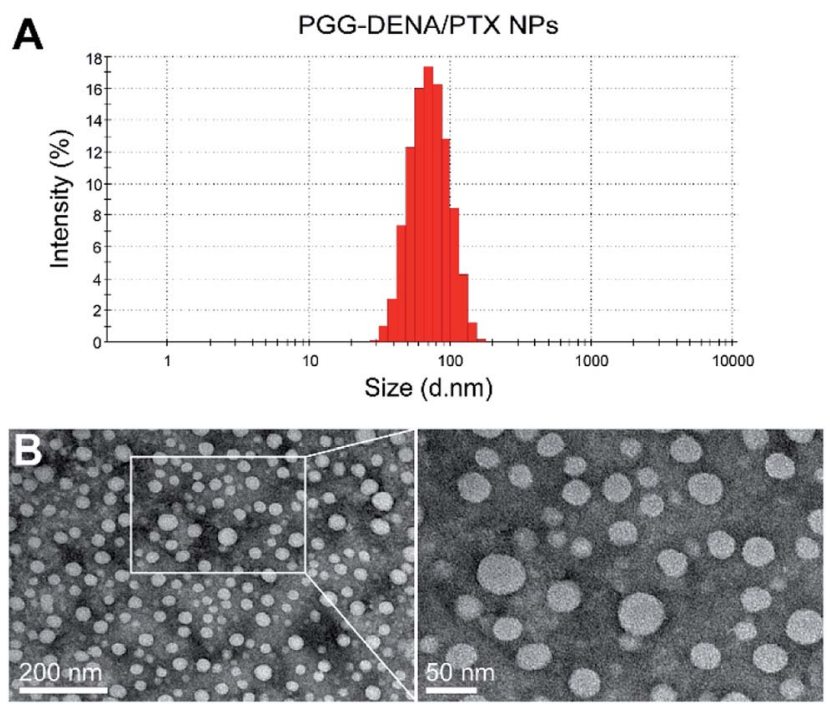

Fig. 5 Particle size and morphology characterization of PGG-DENA/ PTX NPs. DLS analysis (A) and TEM images (B) of PGG-DENA/PTX NPs. The NPs exhibited uniform spherical morphology as displayed in the TEM image. particle size determined by DLS represents their hydrodynamic diameter, whereas that obtained by TEM represents the collapsed micelles after water evaporation. This result is also consistent with previous report. ${ }^{25}$

3.3.2 The long-term stability of PGG-DENA/PTX NPs. The long-term stability of PGG-DENA/PTX NPs was evaluated by the change of particle sizes in 28 days. As shown in Fig. 6, there are no obvious changes, suggesting the good stability of the NPs. This results indicated the high stability of PGG-DENA/PTX NPs, which may be due to the increased compatibility of hydrotropic polymer material with PTX.

3.3.3 Loading capacity of PTX in PGG-DENA NPs. The anticancer drug, PTX, is difficult to encapsulate into traditional micelles because it has the highly hydrophobic property. Thus, we designed "hydrotropic polymer", PGG-DENA NPs, as a carrier for PTX to increase its aqueous solubility. The hydrophobic PTX was easily encapsulated into PGG-DENA NPs using a emulsification-solvent evaporation method due to DENA has a special solubilization mechanism for PTX (aromatic ring buildup and hydrogen bonding). The schematic illustration of PTX loading was showed in Fig. 1. The PTX loading capacity of PGG-DENA/PTX was determined to be $11.7 \%$ (wt $\%$ ) by HPLC determination.

\subsection{In vitro release of PTX from PGG-DENA/PTX NPs}

The PTX release from PGG-DENA/PTX NPs was performed in sodium salicylate $(0.8 \mathrm{M})$ at $37{ }^{\circ} \mathrm{C}$ for $96 \mathrm{~h}$. As shown in Fig. 7, the results showed that the release of PTX from PGG-PTX NPs and PGG-DENA/PTX NPs gradually increased with time. Compared to PGG-PTX NPs (22.62\%), the cumulative release rate of PTX from PGG-DENA/PTX NPs reached $79.10 \%$ at $96 \mathrm{~h}$. PTX release from PGG-DENA/PTX NPs was remarkably higher than that from PGG-PTX NPs. This results indicated that the encapsulated PTX in PGG-DENA/PTX NPs is easier to be released than chemically bonded PTX in PGG-PTX NPs.

\subsection{In vitro cytotoxicity assays}

The cell viability of NCI-H460 cells were evaluated following incubation with PTX, PGG-PTX NPs, PGG-DENA/PTX NPs and

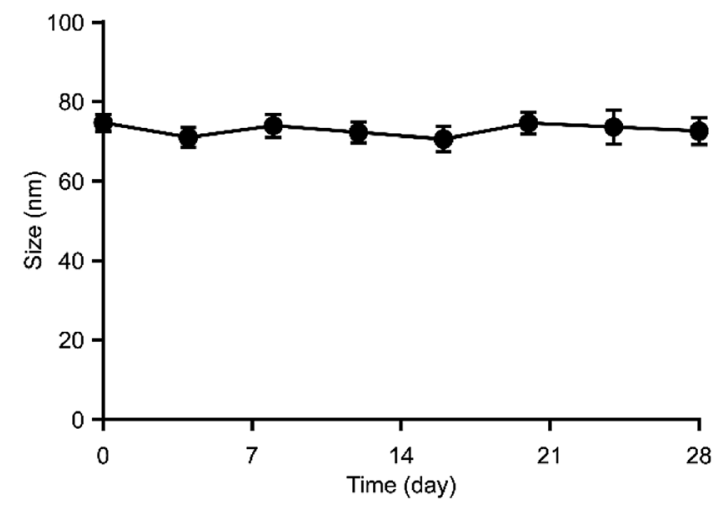

Fig. 6 Stability of PGG-DENA/PTX NPs in PBS solution at $4{ }^{\circ} \mathrm{C}$. PGGDENA/PTX NPs exhibited long-term stability. 


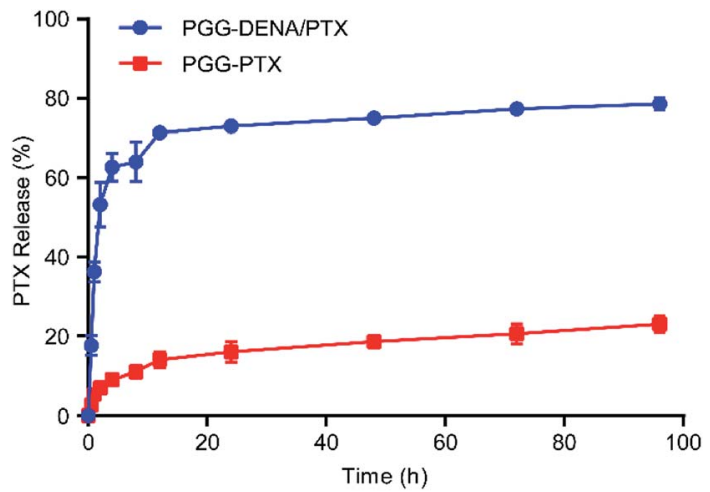

Fig. 7 The kinetics of PTX release from PGG-DENA/PTX NPs and PGG-PTX NPs in sodium salicylate at $37^{\circ} \mathrm{C}(n=3$, bars represent means $\pm S D$ ).

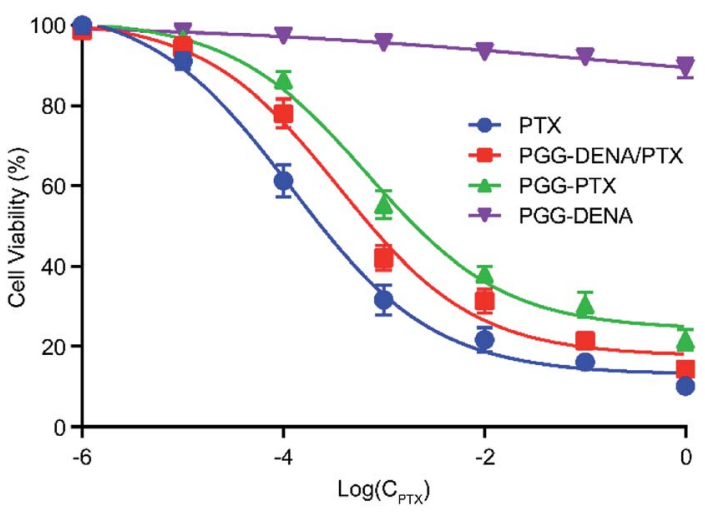

Fig. 8 The cytotoxicity of PTX, PGG-PTX NPs, PGG-DENA/PTX NPs and PGG-DENA on NCl-H460 cells as measured by CCK- 8 Kit. PGGDENA/PTX NPs showed significantly increased cytotoxicity than PGGPTX NPS.

PGG-DENA. As shown in Fig. 8, the results showed that the cell viability of PTX, PGG-DENA/PTX NPs and PGG-PTX NPs decreased gradually as the drug concentration increased. PGGDENA had no obvious cytotoxicity. The $\mathrm{IC}_{50}$ value for PTX, PGGDENA/PTX NPs and PGG-PTX NPs was $0.2304 \mu \mathrm{g} \mathrm{mL}{ }^{-1}, 0.7356$

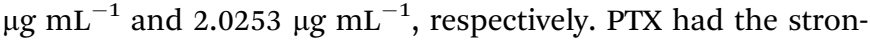
gest cytotoxicity. PGG-DENA/PTX NPs showed significantly increased cytotoxicity than PGG-PTX NPs, which should be resulted from the increased PTX release from PGG-DENA/PTX NPs compared with PGG-PTX NPs (Fig. 7).

\subsection{In vitro cellular uptake}

In order to determine the interaction of PGG-DENA/PTX NPs with the tumor cells, we observed the cellular uptake of NPs by NCI-H460 cell line using CLSM and flow cytometer. As shown in Fig. 9, the percentages of fluorescent cells in PGG-PTX/DiO NPs and PGG-DENA/PTX/DiO NPs were $97.36 \%$ and $96.74 \%$, and the mean fluorescent intensities for them were 183.40 and 177.03, respectively. These data showed that the PGG-PTX/DiO NPs and PGG-DENA/PTX/DiO NPs have similar cellular uptake by NCI-H460 cells, which may be attributed to their similar endocytic pathways.

\subsection{Hemolysis study}

To determine the biocompatibility of PGG-DENA/PTX NPs, a hemolysis study was carried out. Our previous study showed

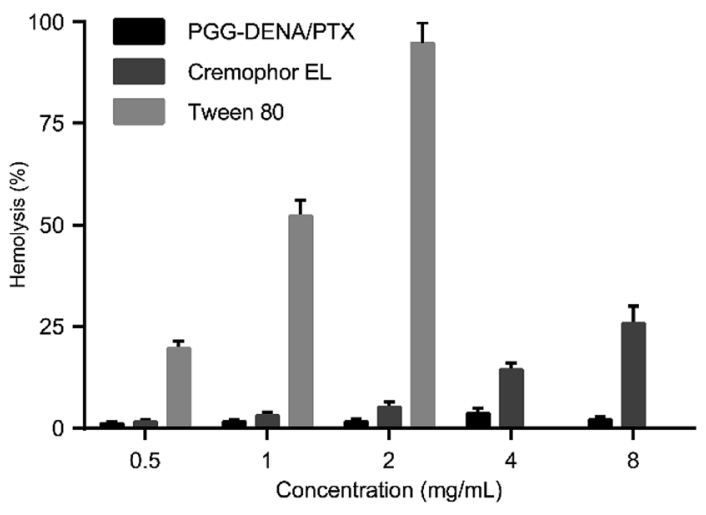

Fig. 10 Hemolysis of red blood cells after incubation with PGGDENA/PTX NPs, Cremophor EL and Tween 80. Compared with Cremophor EL and Tween 80, PGG-DENA/PTX NPs showed almost no hemolytic activity.
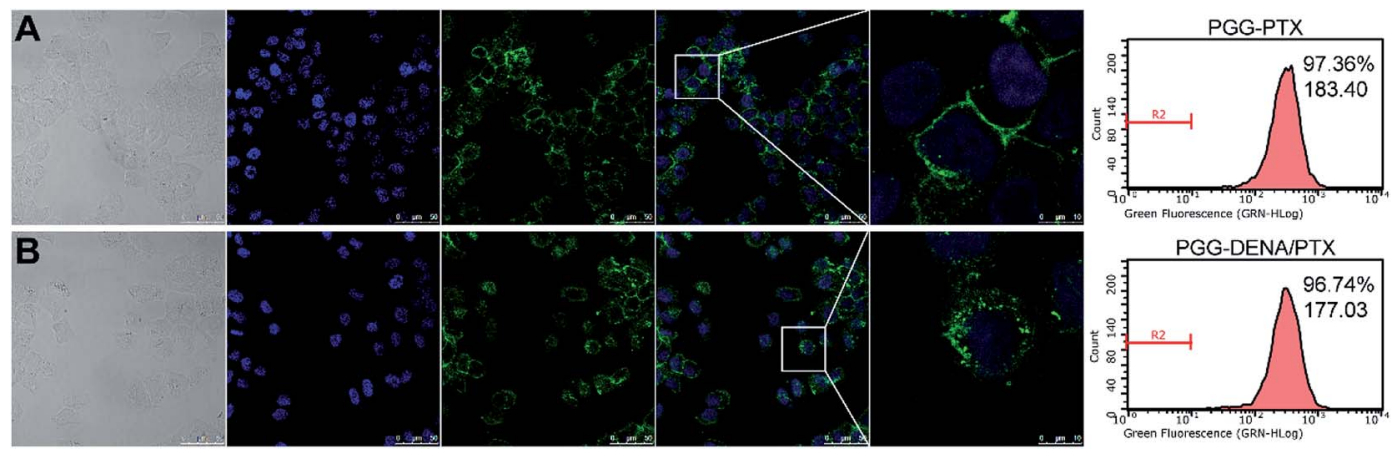

PGG-DENAIPTX

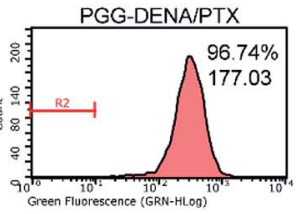

Fig. 9 The CLSM images of cellular uptake and flow cytometry for PGG-PTX/DiO NPs (A) and PGG-DENA/PTX/DiO NPs (B). The numbers in flow cytometry pictures represent percentages of DiO-positive cells and mean of fluorescence intensity, respectively. These data showed that the PGG-PTX/DiO NPs and PGG-DENA/PTX/DiO NPs have similar cellular uptake by NCl-H460 cells. 
that the PGG polymer have good biocompatibility. ${ }^{13}$ As shown in Fig. 10, the surfactants Tween 80 caused significant damage of red blood cells at $2 \mathrm{mg} \mathrm{mL}^{-1}$, and Cremophor EL did not induce substantial hemolysis until reaching $4 \mathrm{mg} \mathrm{mL} \mathrm{m}^{-1}$. By contrast, PGG-DENA/PTX NPs had no hemolytic activity even at high concentrations of $8 \mathrm{mg} \mathrm{mL}^{-1}$. These data suggested that PGGDENA/PTX has good compatibility with erythrocytes and can be administered intravenously.

\section{Conclusions}

In this work, using PTX as the model drug, we conjugated DENA, the hydrotropic agents of PTX, to the backbone of a water-soluble polymer PGG to prepare the "hydrotropic polymer" PGG-DENA. Then by virtue of the hydrotropic effect of DENA group, PTX was successfully encapsulated by PGG-DENA to obtain the hydrotropic polymeric nanoparticles PGG-DENA/ PTX NPs. The results showed that the cumulative release rate of PTX from PGG-DENA/PTX NPs reached $79.10 \%$ at $96 \mathrm{~h}$. In vitro cytotoxicity assays, PGG-DENA/PTX NPs showed significantly increased cytotoxicity than PGG-PTX NPs. The NCI-H460 lung cancer cells have similar cell uptake of PGG-PTX/DiO NPs and PGG-DENA/PTX/DiO NPs. Furthermore, the hemolysis study proved that the PGG-DENA/PTX NPs has good compatibility with erythrocytes. The results indicated that the hydrotropic polymer PGG-DENA was an effective carrier material for PTX.

The molecular structure of the hydrotropic agent is generally composed of an aromatic ring system and an anionic group, in which the aromatic ring moiety interacts with the drug and the anionic group brings a high water solubility. The mechanism of solubilization is much complicated, and the consensus on this can be summarized as the four points: hydrophobic interaction, aromatic ring accumulation, hydrogen bond formation and specific interaction. ${ }^{26}$ Application of hydrotropic agent is a very effective means for the solubilization of insoluble drugs, which can result in the increase of drug solubility by several orders of magnitude. ${ }^{27}$ However, it requires a high concentration of hydrotropic agents for effective solubilization, making it impossible for them to be used in vivo due to their potential toxicity. Thus the current application of hydrotropic agents are mostly limited in the drug solubilization in vitro, such as in drug dissolution test. ${ }^{28}$ Therefore, how to make full use of the solubilization effect of hydrotropic agents, as well as avoid their potential toxicity in vivo, becomes an important scientific issue. Our study here would provide an idea for this issue and expand the use of hydrotropic agents, making them a more effective and practical tool in pharmaceutical science.

The timely and adequate drug release in tumor tissues is a key step to exert the antitumor effect of NDDS. Accordingly, researchers have made great progress in the tumor microenvironment responsive NDDS in recent years, including $\mathrm{pH}$ sensitive,,$^{29,30}$ temperature-sensitive, ${ }^{31}$ redox-sensitive ${ }^{32}$ and enzyme-sensitive, ${ }^{33}$ to improve the drug release behavior. In our study, the cumulative release rate of PTX from PGG-DENA/PTX NPs reached $79.10 \%$ at $96 \mathrm{~h}$, while that of PGG-PTX NPs was only $22.96 \%$ (Fig. 7). This results indicated that the physical encapsulation of drugs might produce more adequate drug release than chemical conjugation does. What is more, PGGDENA/PTX NPs can avoid the side effects of free DENA after administration. We also found that the PGG-DENA/PTX NPs showed significantly increased cytotoxicity than PGG-PTX NPs (Fig. 8), which should be resulted from the increased PTX release from PGG-DENA/PTX NPs compared with PGG-PTX NPs.

In summary, we have successfully developed a PGG-DENA/ PTX NPs delivery system which exhibited a high drug release rate, good biocompatibility and long-term stability. This study provide a solution to increase the compatibility of carrier materials with insoluble drugs, and also may provide an effective way to develop a series of personalized carrier materials suitable for different insoluble drugs.

\section{Conflict of interest}

The authors declare no potential conflicts of interest with respect to the authorship and/or publication of this article.

\section{Acknowledgements}

This work was supported by National Basic Research Program of China (2013CB932500), National Natural Science Foundation of China (60976004), "985” grants of East China Normal University (ECNU).

\section{References}

1 H. K. Ahn, M. Jung, S. J. Sym, D. B. Shin, S. M. Kang, S. Y. Kyung, J. W. Park, S. H. Jeong and E. K. Cho, Cancer Chemother. Pharmacol., 2014, 74, 277-282.

2 X. L. Zhang, Y. X. Huang and S. Li, Ther. Delivery, 2014, 5, 5368.

3 H. Koo, K. H. Min, S. C. Lee, J. H. Park, K. Park, S. Y. Jeong, K. Choi, I. C. Kwon and K. Kim, J. Controlled Release, 2013, 172, 823-831.

4 J. Y. Kim, S. Kim, R. Pinal and K. Park, J. Controlled Release, 2011, 152, 13-20.

5 G. Saravanakumar, K. H. Min, D. S. Min, A. Y. Kim, C. M. Lee, Y. W. Cho, S. C. Lee, K. Kim, S. Y. Jeong, K. Park, J. H. Park and I. C. Kwon, J. Controlled Release, 2009, 140, 210-217.

6 J. J. Booth, M. Omar, S. Abbott and S. Shimizu, Phys. Chem. Chem. Phys., 2015, 17, 8028-8037.

7 K. M. Huh, H. S. Min, S. C. Lee, H. J. Lee, S. Kim and K. Park, J. Controlled Release, 2008, 126, 122-129.

8 X. Gao, Y. Huang, A. M. Makhov, M. Epperly, J. Lu, S. Grab, P. Zhang, L. Rohan, X. Q. Xie, P. Wipf, J. Greenberger and S. Li, Mol. Pharm., 2013, 10, 187-198.

9 S. C. Lee, K. M. Huh, J. Lee, Y. W. Cho, R. E. Galinsky and K. Park, Biomacromolecules, 2007, 8, 202-208.

10 S. Sharma, J. Singh, A. Verma, B. V. Teja, R. P. Shukla, S. K. Singh, V. Sharma, R. Konwar and P. R. Mishra, RSC Adv., 2016, 6, 73083-73095.

11 M. H. Han, H. Zheng, Y. F. Guo, Y. H. Wang, X. Y. Qi and X. T. Wang, RSC Adv., 2016, 6, 45664-45672. 
12 D. Yang, X. Liu, X. Jiang, Y. Liu, W. Ying, H. Wang, H. Bai, W. D. Taylor, Y. Wang, J. P. Clamme, E. Co, P. Chivukula, K. Y. Tsang, Y. Jin and L. Yu, J. Controlled Release, 2012, 161, 124-131.

13 S. Van, S. K. Das, X. Wang, Z. Feng, Y. Jin, Z. Hou, F. Chen, A. Pham, N. Jiang, S. B. Howell and L. Yu, Int. J. Nanomed., 2010, 5, 825-837.

14 D. Yang, L. Yu and S. Van, Cancers, 2010, 3, 17-42.

15 Z. Feng, G. Zhao, L. Yu, D. Gough and S. B. Howell, Cancer Chemother. Pharmacol., 2010, 65, 923-930.

16 D. Yang, S. Van, X. Jiang and L. Yu, Int. J. Nanomed., 2011, 6, 85-91.

17 D. Yang, S. Van, J. Liu, J. Wang, X. Jiang, Y. Wang and L. Yu, Int. J. Nanomed., 2011, 6, 2557-2566.

18 D. Stirland, J. Nichols, S. Miura and Y. Bae, J. Controlled Release, 2013, 172, 1045-1064.

19 X. Li, Y. Ma, X. Zhang and G. Chen, Chin. JMAP, 2011, 28, 740-743.

20 J. H. Kim, Y. S. Kim, K. Park, S. Lee, H. Y. Nam, K. H. Min, H. G. Jo, J. H. Park, K. Choi, S. Y. Jeong, R. W. Park, I. S. Kim, K. Kim and I. C. Kwon, J. Controlled Release, 2008, 127, 41-49.

21 S. Kim, J. Y. Kim, K. M. Huh, G. Acharya and K. Park, J. Controlled Release, 2008, 132, 222-229.

22 Y. Song, R. Guan, F. Lyu, T. Kang, Y. Wu and X. Chen, Mutat. Res., 2014, 769, 113-118.
23 X. Liu, Y. Xie, W. Li, W. Sheng, Y. Li, Z. Tong, H. Ni, C. Huselstein, X. Wang and Y. Chen, Bio-Med. Mater. Eng., 2015, 25, 47-55.

24 J. Lee, S. C. Lee, G. Acharya, C. J. Chang and K. Park, Pharm. Res., 2003, 20, 1022-1030.

25 D. Yang, S. Van, Y. Shu, X. Liu, Y. Ge, X. Jiang, Y. Jin and L. Yu, Int. J. Nanomed., 2012, 7, 581-589.

26 J. Y. Kim, S. Kim, M. Papp, K. Park and R. Pinal, J. Pharm. Sci., 2010, 99, 3953-3965.

27 C. Som, B. Nowack, H. F. Krug and P. Wick, Acc. Chem. Res., 2013, 46, 863-872.

28 B. M. El-Houssieny, E. Z. El-Dein and H. M. El-Messiry, Drug Discoveries Ther., 2014, 8, 178-184.

29 M. Dalela, T. G. Shrivastav, S. Kharbanda and H. Singh, ACS Appl. Mater. Interfaces, 2015, 7, 26530-26548.

30 S. Yin, L. Chang, T. Li, G. Wang, X. Gu and J. Li, RSC Adv., 2016, 6, 105957-105968.

31 Z. Y. Wang, H. Zhang, Y. Yang, X. Y. Xie, Y. F. Yang, Z. Li, Y. Li, W. Gong, F. L. Yu, Z. Yang, M. Y. Li and X. G. Mei, Drug Delivery, 2016, 23, 1222-1231.

32 J. Li, T. Yin, L. Wang, L. Yin, J. Zhou and M. Huo, Int. J. Pharm., 2015, 483, 38-48.

33 N. Li, H. Cai, L. Jiang, J. Hu, A. Bains, J. Hu, Q. Gong, K. Luo and Z. Gu, ACS Appl. Mater. Interfaces, 2017, 9, 6865-6877. 